J. Korean Math. Soc. 52 (2015), No. 4, pp. 663-683

http://dx.doi.org/10.4134/JKMS.2015.52.4.663

\title{
STRUCTURE OF ZERO-DIVISORS IN SKEW POWER SERIES RINGS
}

\author{
Chan Yong Hong, Nam Kyun Kim, and Yang Lee
}

\begin{abstract}
In this note we study the structures of power-serieswise Armendariz rings and IFP rings when they are skewed by ring endomorphisms (or automorphisms). We call such rings skew power-serieswise Armendariz rings and skew IFP rings, respectively. We also investigate relationships among them and construct necessary examples in the process. The results argued in this note can be extended to the ordinary ring theoretic properties of power-serieswise Armendariz rings, IFP rings, and near-related rings.
\end{abstract}

Throughout this paper every ring is an associative ring with identity. Let $\sigma$ be an endomorphism of a ring $R$. We use $R[[x ; \sigma]]$ (resp. $R[x ; \sigma]$ ) to denote the skew power series ring (resp. skew polynomial ring) with an indeterminate $x$ over a ring $R$, subject to the relation $x r=\sigma(r) x$ for $r \in R$. Note that $\sigma(1)=1$ for any skew power series ring (skew polynomial ring) $R[[x ; \sigma]](R[x ; \sigma])$, since $1 x^{n}=x^{n}=x 1 x^{n-1}=\sigma(1) x^{n}$ for any $n \geq 1$ where 1 is the identity of $R$.

Armendariz [2, Lemma 1] proved that whenever polynomials

$$
f(x)=\sum_{i=0}^{m} a_{i} x^{i}, g(x)=\sum_{j=0}^{n} b_{j} x^{j}
$$

over a reduced ring $R$ satisfy $f(x) g(x)=0$, then $a_{i} b_{j}=0$ for all $i, j$. Rege and Chhawchharia [27] called such a ring (not necessarily reduced) Armendariz. The Armendariz condition, and various derivatives described below, have been studied by numerous authors.

According to Kim et al. [16], we say that a $\operatorname{ring} R$ is power-serieswise Armendariz if $a_{i} b_{j}=0$ for all $i, j$ whenever power series $f(x)=\sum_{i=0}^{\infty} a_{i} x^{i}$, $g(x)=\sum_{j=0}^{\infty} b_{j} x^{j}$ in $R[[x]]$ satisfy $f(x) g(x)=0$.

In [12], a ring $R$ with an endomorphism $\sigma$ is called $\sigma$-skew Armendariz if whenever polynomials $f(x)=\sum_{i=0}^{m} a_{i} x^{i}, g(x)=\sum_{j=0}^{n} b_{j} x^{j}$ in $R[x ; \sigma]$ satisfy $f(x) g(x)=0$, then $a_{i} \sigma^{i}\left(b_{j}\right)=0$ for all $i, j$.

Received August 13, 2013; Revised April 7, 2015.

2010 Mathematics Subject Classification. 16S36, 16W20, 16U80.

Key words and phrases. skew power-serieswise Armendariz ring, skew IFP ring. 
Cortes [9, Definition 2.2] extended this definition to a skew power-serieswise Armendariz ring as follows, and then studied the zip property of a skew power series ring over a zip ring under the ring. (Recall that a ring $\mathrm{R}$ right zip if the right annihilator $r_{R}(X)$ of a subset $X$ of $R$ is zero, then $r_{R}(Y)=0$ for a finite subset $Y \subseteq X$.) A ring $R$ with an endomorphism $\sigma$ satisfies $S A \mathscr{L}^{\prime}$ if whenever power series $f(x)=\sum_{i=0}^{\infty} a_{i} x^{i}, g(x)=\sum_{j=0}^{\infty} b_{j} x^{j}$ in $R[[x ; \sigma]]$ satisfy $f(x) g(x)=0$, then $a_{i} \sigma^{i}\left(b_{j}\right)=0$ for all $i, j$. However, we call the ring $\sigma$-skew power-serieswise Armendariz.

According to Baser et al. [3, Definition 3.1], a ring $R$ with an endomorphism $\sigma$ is called $\sigma$-sps Armendariz if whenever power series $f(x)=\sum_{i=0}^{\infty} a_{i} x^{i}, g(x)=$ $\sum_{j=0}^{\infty} b_{j} x^{j}$ in $R[[x ; \sigma]]$ satisfy $f(x) g(x)=0$, then $a_{i} b_{j}=0$ for all $i, j$. If we use the methods of [14, Theorem 1.8], we get the fact that $\sigma$-sps Armendariz rings are $\sigma$-skew power-serieswise Armendariz, but the converse is not true (see $[14$, Example 1.9]).

Krempa [17] called an endomorphism $\sigma$ of a ring $R$ rigid if $a \sigma(a)=0$ implies $a=0$ for $a \in R$. We call a ring $R \sigma$-rigid if there exists a rigid endomorphism $\sigma$ of $R$. Note that any rigid endomorphism of a ring is a monomorphism and $\sigma$-rigid rings are reduced rings by [12, Proposition 3]. Using this result, we can easily check that $\sigma$-rigid rings are $\sigma$-skew power-serieswise Armendariz.

A ring $R$ is called to satisfy the insertion-of-factors-property (simply, an IFP ring) if $a b=0$ implies $a R b=0$ for $a, b \in R$ [4]. Narbonne [22] and Shin [28] used the terms semicommutative and $S I$ for the IFP, respectively. A ring $R$ is usually called abelian if every idempotent element is central. IFP rings are obviously abelian. These conditions have the following relationships:

$$
\text { reduced } \Rightarrow \text { power-serieswise Armendariz } \Rightarrow \text { IFP } \Rightarrow \text { abelian }
$$

by [16, Lemma 2.3] and [28, Lemma 2.7]. However, without identity, an IFP ring need not be abelian as can be seen by $T=\left(\begin{array}{cc}S & S \\ 0 & 0\end{array}\right)$ over a domain $S$ with identity.

In this note we will observe the previously mentioned conditions when they are equipped with an endomorphism (automorphism), and we study relationships between them.

\section{Skew power-serieswise Armendariz rings}

In this section, we study skew power-serieswise Armendariz rings which extend power-serieswise Armendariz rings and skew Armendariz rings, etc. We first note that $\sigma$-rigid rings are reduced rings by [12, Proposition 3]. Using this result, we can easily check that $\sigma$-rigid rings are $\sigma$-skew power-serieswise Armendariz. Moreover, we have the following result which gives a very short proof to Matczuk's result [21, Theorem A] and also contains a result [3, Theorem 3.3(1)].

Theorem 1.1. Let $\sigma$ be an endomorphism of a ring $R$. Then the following conditions are equivalent: 
(1) $R$ is $\sigma$-rigid.

(2) $\sigma$ is injective, $R$ is reduced and $\sigma$-skew power-serieswise Armendariz.

(3) $\sigma$ is injective, $R$ is reduced and $\sigma$-skew Armendariz.

(4) $R[[x ; \sigma]]$ is reduced.

(5) $R[x ; \sigma]$ is reduced.

Proof. (1) $\Leftrightarrow(4) \Leftrightarrow(5)$ follow from [12, Proposition 3] and (1) $\Rightarrow(2)$ is routine. $(3) \Rightarrow(1)$ : Suppose $a \sigma(a)=0$ for $a \in R$. Since $R$ is reduced, $\sigma(a) a=0$. Thus $\sigma(a)(1-\sigma(a) x)(1+\sigma(a) x) a=\sigma(a) a=0$. Since $R$ is $\sigma$-skew Armendariz, $0=\sigma(a) \sigma(a) \sigma(a)=\sigma\left(a^{3}\right)$ and so $a^{3}=0$, entailing $a=0$.

Referring to [12, Proposition 3] or by Theorem 1.1, a ring $R$ is $\sigma$-rigid if and only if $R[[x ; \sigma]]$ is reduced. Then $\sigma$-rigid rings are $\sigma$-skew power-serieswise Armendariz. However, the converse is not true. For example, the ring $R$ in [12, Example 1] is not $\sigma$-rigid, but we can get that $R$ is $\sigma$-skew power-serieswise Armendariz by the same method.

It is clear that $\sigma$-skew power-serieswise Armendariz rings are $\sigma$-skew $\mathrm{Ar}$ mendariz rings. However, the converse is also not true by the following result which is a generalization to the skew setting of a special case of [1, Theorem 4.7].

Let $K$ be a field and $R_{1}, R_{2}$ be $K$-algebras. Use $R_{1} *_{K} R_{2}$ to denote the ring coproduct of $R_{1}$ and $R_{2}$ (see Antoine [1] and Bergman [5,6] for details). Given a ring $R, U(R)$ means the group of units in $R$.

Theorem 1.2. Let $K$ be a field and $A$ be a $K$-algebra. Let $C=K[c]$ be the polynomial ring with an indeterminate $c$ over $K$, and $I$ be the ideal of $C$ generated by $c^{2}$. Set $B=C / I$ and $R=A *_{K} B$. Let $\alpha$ be an automorphism of $A$ and $\sigma$ be the extended automorphism of $R$ defined by $\sigma(a)=\alpha(a)$ for $a \in A$ and $\sigma(c+I)=c+I$. Then $R$ is $\sigma$-skew Armendariz if and only if $A$ is a domain and $U(A)=K \backslash\{0\}$.

Proof. Suppose that $A$ is a domain and $U(A)=K \backslash\{0\}$. We identify $a_{i}$ with $a_{i}+I$ for simplicity. Let $0 \neq f(x)=\sum_{i=0}^{m} a_{i} x^{i}, 0 \neq g(x)=\sum_{j=0}^{n} b_{j} x^{j} \in R[x ; \sigma]$ such that $f(x) g(x)=0$. Then $f(x)$ and $g(x)$ can be expressed by the following forms:

$$
f(x)=f_{0}+f_{1} c+c f_{2}+c f_{3} c \text { and } g(x)=g_{0}+g_{1} c+c g_{2}+c g_{3} c,
$$

where $f_{i}, g_{i} \in R[x ; \sigma]$ and every sum-factor of coefficients of $f_{i}$ 's and $g_{i}$ 's does not start by $c$ and does not end by $c$. In the following computation we will use freely the condition that $A$ is a domain and $U(A)=K \backslash\{0\}$. Considering the normal form of the elements in a coproduct of rings, every sum-factor of an element in $R$ is one of the following forms:

$$
a, a_{1} c a_{2}, a_{1} c a_{2} c a_{3}, \ldots, a_{1} c a_{2} c \cdots a_{t-1} c a_{t},
$$

where $a, a_{i} \in A$ for all $i$, and $t \geq 2$. Note that $\sigma\left(a_{1} c a_{2} c \cdots a_{t-1} c a_{t}\right)=$ $\sigma\left(a_{1}\right) c \sigma\left(a_{2}\right) c \cdots \sigma\left(a_{t-1}\right) c \sigma\left(a_{t}\right)$. This yields that $\sigma(r) \neq 0$ if and only if $r \neq 0$ for $r \in R$. From $f(x) g(x)=0$, we have $f_{0}=0$ or $g_{0}=0$. 
Case 1. $f_{0} \neq 0$ and $g_{0}=0$.

Note

$0=f(x) g(x)=f_{0} g_{1} c+f_{0} c g_{2}+f_{0} c g_{3} c+f_{1} c g_{1} c+c f_{2} g_{1} c+c f_{2} c g_{2}+c f_{2} c g_{3} c+c f_{3} c g_{1} c$.

Since $f_{0} g_{1} c$ is unique in the expansion of $f(x) g(x)$, we must have $f_{0} g_{1} c=0$ and it follows that $g_{1} c=0$. Hence

$$
0=f(x) g(x)=f_{0} c g_{2}+f_{0} c g_{3} c+c f_{2} c g_{2}+c f_{2} c g_{3} c .
$$

This also yields $c g_{2}=0$ and $c g_{3} c=0$. Consequently $g(x)=0$, a contradiction.

Case 2. $f_{0}=0$ and $g_{0} \neq 0$.

Note

$0=f(x) g(x)=f_{1} c g_{0}+f_{1} c g_{1} c+c f_{2} g_{0}+c f_{2} g_{1} c+c f_{2} c g_{2}+c f_{2} c g_{3} c+c f_{3} c g_{0}+c f_{3} c g_{1} c$.

Since $f_{1} c g_{0}$ is unique in the expansion of $f(x) g(x)$, we must have $f_{1} c g_{0}=0$.

Thus, $f_{1} c=0$ and

$$
0=f(x) g(x)=c f_{2} g_{0}+c f_{2} g_{1} c+c f_{2} c g_{2}+c f_{2} c g_{3} c+c f_{3} c g_{0}+c f_{3} c g_{1} c .
$$

Note that $c f_{2} g_{0}$ is also unique in the expansion, and so $c f_{2}=0$. This yields $c f_{3} c=0$ and we have that $f(x)=0$, a contradiction.

Case 3. $f_{0}=0$ and $g_{0}=0$.

Note $f(x)=f_{1} c+c f_{2}+c f_{3} c, g(x)=g_{1} c+c g_{2}+c g_{3} c$ and

$$
0=f(x) g(x)=f_{1} c g_{1} c+c f_{2} c g_{2}+c f_{2} g_{1} c+c f_{2} c g_{3} c+c f_{3} c g_{1} c .
$$

In this case, we have that $f_{1} c g_{1} c$ is the unique in the expansion of $f(x) g(x)$, we must have $f_{1} c g_{1} c=0$. Similarly $c f_{2} c g_{2}=0$. Hence "either $f_{1} c=0$ or $g_{1} c=0$ " and "either $c f_{2}=0$ or $c g_{2}=0$ ". Consequently, we have the following three cases.

Subcase 3-1. $f_{1} c=0$ and $c f_{2}=0$.

Note that $f(x)=c f_{3} c, g(x)=g_{1} c+c g_{2}+c g_{3} c$ and $0=f(x) g(x)=c f_{3} c g_{1} c$. Since $c f_{3} c \neq 0$, we get $g_{1} c=0$ and so $g(x)=c g_{2}+c g_{3} c$.

Subcase 3-2. $f_{1} c \neq 0$ and $c f_{2}=0$.

We have that $f(x)=f_{1} c+c f_{3} c, g(x)=g_{1} c+c g_{2}+c g_{3} c$ and

$$
0=f(x) g(x)=f_{1} c g_{1} c+c f_{3} c g_{1} c .
$$

Since $f_{1} c g_{1} c$ and $c f_{3} c g_{1} c$ are both unique in the expansion of $f(x) g(x)$, we get $f_{1} c g_{1} c=0$ and $c f_{3} c g_{1} c=0$. Since $f_{1} c \neq 0$ and then $g_{1} c=0$. Thus, $g(x)=c g_{2}+c g_{3} c$.

Subcase 3-3. $f_{1} c=0$ and $c f_{2} \neq 0$.

Note that $f(x)=c f_{2}+c f_{3} c, g(x)=g_{1} c+c g_{2}+c g_{3} c$ and

$$
0=f(x) g(x)=c f_{2} c g_{2}+c f_{2} g_{1} c+c f_{2} c g_{3} c+c f_{3} c g_{1} c .
$$


Since $c f_{2} c g_{2}$ is unique in the expansion of $f(x) g(x)$, we get $c f_{2} c g_{2}=0$. By the fact that $c f_{2} \neq 0$ we have that $c g_{2}=0$. Thus, $g(x)=g_{1} c+c g_{3} c$ and

$$
f(x) g(x)=c f_{2} g_{1} c+c f_{2} c g_{3} c+c f_{3} c g_{1} c .
$$

In the right hand side of the preceding equality, $c f_{2} g_{1} c$ is also unique and we get $g_{1} c=0$, which implies that $g(x)=c g_{3} c$. Now we have $0=f(x) g(x)=c f_{2} c g_{3} c$. Since $c f_{2} \neq 0, c g_{3} c=0$ we have that $g(x)=0$, a contradiction.

Summarizing the computations, we have $f(x)=h(x) c$ and $g(x)=c k(x)$ for some $h(x), k(x) \in R[x ; \sigma]$ whenever $f(x) g(x)=0$. This implies that

$$
a_{i} \sigma^{i}\left(b_{j}\right)=a_{i}^{\prime} c \sigma^{i}\left(c b_{j}^{\prime}\right)=a_{i}^{\prime} c c \sigma^{i}\left(b_{j}^{\prime}\right)=0
$$

for all $i$ and $j$, where $a_{i}=a_{i}^{\prime} c$ and $b_{j}=c b_{j}^{\prime}$ for some $a_{i}^{\prime}, b_{j}^{\prime} \in R$. So $R$ is $\sigma$-skew Armendariz.

For the proof of the converse of the theorem, we apply the method in the proof of [1, Theorem 4.7]. Let $R$ be $\sigma$-skew Armendariz and assume on the contrary that

$A$ is not a domain or $U(A) \supsetneqq K \backslash\{0\}$.

Suppose that there exist $\alpha, \beta \in A \backslash\{0\}$ such that $\alpha \beta=0$. Set $a=\beta c \alpha$. Then $0 \neq a \in R$ and $a^{2}=0$. Consider two nonzero polynomials

$$
f(x)=a+a c x \text { and } g(x)=a c-c \sigma(a) c x
$$

in $R[x ; \sigma]$. Then

$$
\begin{aligned}
f(x) g(x) & =(a+a c x)(a c-c \sigma(a) c x) \\
& =a^{2} c-a c \sigma(a) c x+a c \sigma(a) c x-a c^{2} \sigma^{2}(a) c x^{2}=0,
\end{aligned}
$$

but $a c \sigma(a) c$ is nonzero. This implies that $R$ is not a $\sigma$-skew Armendariz ring.

Next let $u \in U(A) \backslash K$, and consider two nonzero polynomials

$$
f(x)=c u^{-1}-c u^{-1} c x \text { and } g(x)=u c+c \sigma(u) c x
$$

in $R[x ; \sigma]$. Then

$$
\begin{aligned}
f(x) g(x) & =\left(c u^{-1}-c u^{-1} c x\right)(u c+c \sigma(u) c x) \\
& =c^{2}+c u^{-1} c \sigma(u) c x-c u^{-1} c \sigma(u) c x-c u^{-1} c^{2} \sigma^{2}(u) c x^{2}=0
\end{aligned}
$$

but $c u^{-1} c \sigma(u) c$ is nonzero, which implies that $R$ is not a $\sigma$-skew Armendariz ring.

Using Theorem 1.2, we can always construct a $\sigma$-skew Armendariz ring but not $\sigma$-skew power-serieswise Armendariz whenever a field is given.

Example 1.3. Let $K$ be a field and $A$ be a $K$-algebra such that $\operatorname{dim}_{K} A \geq 2$ and $U(A)=K \backslash\{0\}$ (e.g., the free algebra generated by noncommuting indeterminates $a, b$ over $K)$. Let $C=K[c]$ be the polynomial ring with an indeterminate $c$ over $K$, and $I$ be the ideal of $C$ generated by $c^{2}$, set $B=C / I$ and $R=A *_{K} B, \alpha$ an automorphism of $A$ and $\sigma$ the extended automorphism 
of $R$ defined by $\sigma(a)=\alpha(a)$ for $a \in A$ and $\sigma(c+I)=c+I$. Then $R$ is $\sigma$-skew Armendariz by Theorem 1.2. Next consider the product of nonzero power series

$$
c(1-a x)\left(1+a x+a \sigma(a) x^{2}+a \sigma(a) \sigma^{2}(a) x^{3}+\cdots\right) c=c^{2}=0
$$

for $a \in A \backslash K$ where $c(1-a x)=c-c a x,\left(1+a x+a \sigma(a) x^{2}+a \sigma(a) \sigma^{2}(a) x^{3}+\cdots\right) c=$ $c+a c x+a \sigma(a) c x^{2}+a \sigma(a) \sigma^{2}(a) c x^{3}+\cdots \in R[[x ; \sigma]]$. Since $c a c \neq 0$ we have that $R$ is not $\sigma$-skew power-serieswise Armendariz.

We now provide a lemma which is very useful to study $\sigma$-skew powerserieswise Armendariz rings, and which is compared with [16, Lemma 2.3]. If $\sigma$ is an endomorphism of $R$, then $\sigma$ induces an endomorphism $\bar{\sigma}$ on $R[[x ; \sigma]]$ $(R[x ; \sigma])$ by $\bar{\sigma}\left(\sum a_{i} x^{i}\right)=\sum \sigma\left(a_{i}\right) x^{i}$.

Lemma 1.4. Suppose $R$ is a $\sigma$-skew power-serieswise Armendariz ring with an endomorphism $\sigma$. Then we have the following:

(1) If $f_{1}(x), \ldots, f_{n}(x) \in R[[x ; \sigma]]$ such that $f_{1}(x) f_{2}(x) \cdots f_{n}(x)=0$, then

$$
a_{i_{1}} \sigma^{i_{1}}\left(a_{j_{2}}\right) \cdots \sigma^{s_{n-1}+\cdots+j_{2}+i_{1}}\left(a_{t_{n}}\right)=0 \text {, }
$$

where $a_{i_{1}}, a_{j_{2}}, \ldots, a_{s_{n-1}} a_{t_{n}}$ are arbitrary coefficients of $f_{1}(x), f_{2}(x), \ldots$, $f_{n-1}(x), f_{n}(x)$, respectively.

(2) For $a, b \in R, a b=0$ implies $a R \sigma^{n}(b)=0$ for all $n \geq 1$.

(3) For $f(x), g(x) \in R[[x ; \sigma]], f(x) g(x)=0$ implies $f(x) I g(x)=0$, where $I$ is a left (or right) ideal of $R[[x ; \sigma]]$ generated by $x$.

Proof. (1) Suppose $f_{1}(x) f_{2}(x) \cdots f_{n}(x)=0$. By assumption we have that $a_{i_{1}} \sigma^{i_{1}}(b)=0$ for any coefficient $a_{i_{1}}$ of $f_{1}(x)$ and any coefficient $b$ of $f_{2}(x) \cdots f_{n}(x)$. This implies $a_{i_{1}} \bar{\sigma}^{i_{1}}\left(f_{2}(x) \cdots f_{n}(x)\right)=0$, and so $\left(a_{i_{1}} \sigma^{i_{1}}\left(a_{0_{2}}\right)+\left(a_{i_{1}} \sigma^{i_{1}}\left(a_{1_{2}}\right)\right) x+\right.$ $\cdots) \bar{\sigma}^{i_{1}}\left(f_{3}(x) \cdots f_{n}(x)\right)=0$. Thus $\left(a_{i_{1}} \sigma^{i_{1}}\left(a_{j_{2}}\right)\right) \sigma^{j_{2}}(d)=0$ for any coefficient $a_{j_{2}}$ of $f_{2}(x)$ and any coefficient $d$ of $\bar{\sigma}^{i_{1}}\left(f_{3}(x) \cdots f_{n}(x)\right)$. Note that $d$ is of the form $\sigma^{i_{1}}(c)$ for some coefficient $c$ of $f_{3}(x) \cdots f_{n}(x)$. This implies $a_{i_{1}} \sigma^{i_{1}}\left(a_{j_{2}}\right) \bar{\sigma}^{j_{2}+i_{1}}\left(f_{3}(x) \cdots f_{n}(x)\right)=0$, and it follows that

$\left(a_{i_{1}} \sigma^{i_{1}}\left(a_{j_{2}}\right) \sigma^{j_{2}+i_{1}}\left(a_{0_{3}}\right)+\left(a_{i_{1}} \sigma^{i_{1}}\left(a_{j_{2}}\right) \sigma^{j_{2}+i_{1}}\left(a_{1_{3}}\right)\right) x+\cdots\right) \bar{\sigma}^{j_{2}+i_{1}}\left(f_{4}(x) \cdots f_{n}(x)\right)$ $=0$.

Continuing this process, we inductively obtain

$$
a_{i_{1}} \sigma^{i_{1}}\left(a_{j_{2}}\right) \sigma^{j_{2}+i_{1}}\left(a_{k_{3}}\right) \cdots \sigma^{s_{n-1}+\cdots+j_{2}+i_{1}}\left(a_{t_{n}}\right)=0,
$$

where $a_{k_{3}}$ and $a_{t_{n}}$ are arbitrary coefficients of $f_{3}(x)$ and $f_{n}(x)$, respectively.

(2) Suppose that $a b=0$ for $a, b \in R$. Then, for any integer $l \geq 1$ and $r \in R$, we have

$$
\begin{aligned}
0 & =a b \\
& =a\left(1-r x^{l}\right)\left(1+r x^{l}+r \sigma^{l}(r) x^{2 l}+r \sigma^{l}(r) \sigma^{2 l}(r) x^{3 l}+\cdots\right) b \\
& =\left(a-a r x^{l}\right)\left(b+r \sigma^{l}(b) x^{l}+r \sigma^{l}(r) \sigma^{2 l}(b) x^{2 l}+r \sigma^{l}(r) \sigma^{2 l}(r) \sigma^{3 l}(b) x^{3 l}+\cdots\right) .
\end{aligned}
$$

Since $R$ is $\sigma$-skew power-serieswise Armendariz, we have $\operatorname{ar}^{l}(b)=0$. Thus, $a R \sigma^{l}(b)=0$. 
(3) Let $f(x)=\sum_{i=0}^{\infty} a_{i} x^{i}, g(x)=\sum_{j=0}^{\infty} b_{j} x^{j} \in R[[x ; \sigma]]$ such that $f(x) g(x)=$ 0 . Then $a_{i} \sigma^{i}\left(b_{j}\right)=0$ for all $i, j$. From $(2), a_{i} R \sigma^{n+i}\left(b_{j}\right)=0$ for all $i, j$ and $n \geq 1$. Thus for any $r \in R$ and integer $t \geq 1$, commutative ring $R$, see [10, Examples 1, 2, and 3].

$$
\begin{aligned}
& f(x) r x^{t} g(x) \\
= & \left(\sum_{i=0}^{\infty} a_{i} x^{i}\right) r x^{t}\left(\sum_{j=0}^{\infty} b_{j} x^{j}\right) \\
= & a_{0} r \sigma^{t}\left(b_{0}\right)+\left(a_{0} r \sigma^{t}\left(b_{1}\right)+a_{1} \sigma(r) \sigma^{t+1}\left(b_{0}\right)\right) x+\cdots=0,
\end{aligned}
$$

completing the proof.

It is well known that a polynomial $f(x)$ over a commutative ring $R$ is nilpotent if and only if each coefficient of $f(x)$ is nilpotent, and that $f(x)$ is a zero divisor in $R[x]$ if and only if $f(x)$ is annihilated by a nonzero element of $R$. However these results are not true in the power series ring $R[[x]]$ over a commutative ring $R$, see [10, Examples 1, 2, and 3].

Proposition 1.5. Let $R$ be a $\sigma$-skew power-serieswise Armendariz ring with an endomorphism $\sigma$. For nonzero elements $f(x), g(x) \in R[[x ; \sigma]]$, if $f(x) g(x)=0$, then $f(x) c=0$ for some $0 \neq c \in R$.

Proof. Let $f(x)=\sum_{i=0}^{\infty} a_{i} x^{i}$ and $g(x)=\sum_{j=0}^{\infty} b_{j} x^{j}$ be nonzero elements in $R[[x ; \sigma]]$ with $f(x) g(x)=0$. Then by Lemma 1.4(3), $f(x) x g(x)=0$. If $x g(x) \neq$ 0 , then there exists $i$ such that $\sigma\left(b_{i}\right) \neq 0$. Since $f(x)(x g(x))=0, a_{k} \sigma^{k}\left(\sigma\left(b_{i}\right)\right)=$ 0 for all $k \geq 0$. Thus $f(x) \sigma\left(b_{i}\right)=0$. Again applying $x g(x)=0$, then $\sigma\left(b_{k}\right)=0$ for all $k \geq 0$. Since $g(x) \neq 0$, there exists $i$ such that $b_{i} \neq 0$. By the fact that $f(x) g(x)=0$ we have that $a_{t} \sigma^{t}\left(b_{i}\right)=0$ for all $t \geq 0$. So $f(x) b_{i}=0$.

Let $\sigma$ be an endomorphism of a ring $R$. An element $a$ of $R$ is called $\sigma$ nilpotent if for any integer $l \geq 1$, there exists a positive integer $m=m(l)$, depending on $l$, such that $a \sigma^{l}(a) \sigma^{2 l}(a) \cdots \sigma^{(m-1) l}(a)=0$. Equivalently, the elements $a x^{l} \in R[x ; \sigma]$ are nilpotent for each integer $l \geq 1$ (cf. [13], [24], [26], etc.). We recall that a subset $S$ of a ring $R$ is called $\sigma$-nilpotent if for any integer $l \geq 1$, there exists a positive integer $m=m(l)$, depending on $l$, such that $S \sigma^{l}(S) \sigma^{2 l}(S) \cdots \sigma^{(m-1) l}(S)=0$ (cf. [13]).

Theorem 1.6. Let $R$ be a $\sigma$-skew power-serieswise Armendariz ring with an endomorphism $\sigma$ and $f(x)=\sum_{i=0}^{\infty} a_{i} x^{i} \in R[[x ; \sigma]]$. Then the following statements are equivalent:

(1) $f(x)$ is $\bar{\sigma}$-nilpotent;

(2) For any $l \geq 1$, there exists $n=n(l) \geq 1$ such that

$$
a_{s_{1}} \sigma^{l+s_{1}}\left(a_{s_{2}}\right) \sigma^{2 l+\left(s_{1}+s_{2}\right)}\left(a_{s_{3}}\right) \cdots \sigma^{(n-1) l+\left(s_{1}+s_{2}+\cdots+s_{n-1}\right)}\left(a_{s_{n}}\right)=0
$$

for any choice of coefficients $a_{s_{j}}$ 's of $f(x)(j=1,2, \ldots, n)$.

In this case, each ideal $R a_{i} R$ is $\sigma$-nilpotent. 
Proof. Let $f(x)$ be $\bar{\sigma}$-nilpotent. Then for any integer $l \geq 1$, there exists a positive integer $n=n(l)$ such that

$$
\begin{aligned}
0 & =f(x) \bar{\sigma}^{l}(f(x)) \cdots \bar{\sigma}^{(n-1) l}(f(x)) \\
& =\left(a_{0}+a_{1} x+\cdots\right)\left(\sigma^{l}\left(a_{0}\right)+\sigma^{l}\left(a_{1}\right) x+\cdots\right) \cdots\left(\sigma^{(n-1) l}\left(a_{0}\right)+\sigma^{(n-1) l}\left(a_{1}\right) x+\cdots\right) .
\end{aligned}
$$

By Lemma 1.4(1), we have

$$
a_{s_{1}} \sigma^{l+s_{1}}\left(a_{s_{2}}\right) \sigma^{2 l+\left(s_{1}+s_{2}\right)}\left(a_{s_{3}}\right) \cdots \sigma^{(n-1) l+\left(s_{1}+s_{2}+\cdots+s_{n-1}\right)}\left(a_{s_{n}}\right)=0
$$

for any choice of coefficients $a_{s_{j}}$ 's of $f(x)(j=1,2, \ldots, n)$. Then, by Lemma $1.4(2)$, we have

$$
\begin{aligned}
& R a_{s_{1}} R \sigma^{l+s_{1}+t_{1}}\left(a_{s_{2}}\right) R \sigma^{2 l+\left(s_{1}+s_{2}\right)+\left(t_{1}+t_{2}\right)}\left(a_{s_{3}}\right) \\
& \cdots R \sigma^{(n-1) l+\sum_{i=0}^{n-1}\left(s_{i}+t_{i}\right)}\left(a_{s_{n}}\right) R=0
\end{aligned}
$$

for any positive integer $t_{k}(k=1,2, \ldots, n-1)$. This yields

$$
\begin{aligned}
& R a_{s_{1}} R \sigma^{l+s_{1}+t_{1}}\left(R a_{s_{2}} R\right) \sigma^{2 l+\left(s_{1}+s_{2}\right)+\left(t_{1}+t_{2}\right)}\left(R a_{s_{3}} R\right) \\
& \cdots \sigma^{(n-1) l+\sum_{i=0}^{n-1}\left(s_{i}+t_{i}\right)}\left(R a_{s_{n}} R\right)=0 .
\end{aligned}
$$

Next let $a_{s_{1}}=\cdots=a_{s_{n}}=a_{i}$. Then

$$
\begin{aligned}
& \left(R a_{i} R\right) R \sigma^{l+i+t_{1}}\left(R a_{i} R\right) R \sigma^{2(l+i)+\left(t_{1}+t_{2}\right)}\left(R a_{i} R\right) R \\
& \cdots \sigma^{(n-1)(l+i)+\sum_{i=0}^{n-1} t_{i}}\left(R a_{i} R\right) R=0 .
\end{aligned}
$$

Since each $t_{k}$ runs over all positive integers, we can obtain

$$
\begin{aligned}
0= & \left(R a_{i} R\right) \sigma^{l}\left(R a_{i} R\right) \cdots \sigma^{\left(\alpha_{1}-1\right) l}\left(R a_{i} R\right) \sigma^{\alpha_{1} l}\left(R a_{i} R\right) \sigma^{\left(\alpha_{1}+1\right) l}\left(R a_{i} R\right) \\
& \cdots \sigma^{\left(\alpha_{2}-1\right) l}\left(R a_{i} R\right) \sigma^{\alpha_{2} l}\left(R a_{i} R\right) \sigma^{\left(\alpha_{2}+1\right) l}\left(R a_{i} R\right) \\
& \cdots \sigma^{\left(\alpha_{n-1}-1\right) l}\left(R a_{i} R\right) \sigma^{\alpha_{n-1} l}\left(R a_{i} R\right)
\end{aligned}
$$

by inserting necessary subsets of the form $\sigma^{u}\left(R a_{i} R\right.$ ) (with $u \geq 1$ ) in place of $R$ 's, where we let $t_{k}$ 's such that $l+i+t_{1}=\alpha_{1}, 2(l+i)+\left(t_{1}+t_{2}\right)=\alpha_{2}, \ldots$, $(n-1)(l+i)+\sum_{i=0}^{n-1} t_{i}=\alpha_{n-1}$.

The proof of the converse is obvious.

In the proof of Theorem 1.6, we have that $R a_{i} R$ is $\sigma$-nilpotent. A related example, in case of $\sigma$ being an identity map, can be found in [10]. The condition " $R$ is a $\sigma$-skew power-serieswise Armendariz ring" in Proposition 1.5 is essential as the following example shows.

Example 1.7. Let $D$ be a commutative domain, $X=\left\{t_{j} \mid j \in \mathbb{Z}\right\}$ a set of commuting indeterminates over $D$ and $F$ is the quotient field of $D[X]$ and $\alpha$ an automorphism of $F$ defined by $t_{i} \mapsto t_{i+1}$. We consider $A=F\{a, b, c\}$, the free algebra of polynomials with zero constant terms in noncommuting indeterminates $a, b, c$ over $F$, and $I$ be an ideal of $F+A$ generated by $c c, a c, c r c$ with $r \in A$. Next we define $R=\frac{F+A}{I}$ and an automorphism $\sigma$ of $R$ by $\sigma\left(\sum_{\text {finite }} s Y\right) \rightarrow \sum_{\text {finite }} \alpha(s) Y$, where $s \in F$ and $Y$ is a finite product of $a, b, c$ 's. 
Identify $f$ with $f+I$ for $f \in F+A$ for simplicity. Consider the equality $(a-a b x)\left(c+b c x+b^{2} c x^{2}+\cdots+b^{n} c x^{n}+\cdots\right)=a(1-b x)\left(1+b x+b^{2} x^{2}+\cdots\right) c=$ $a c=0$ with $1-b x, 1+b x+b^{2} x^{2}+\cdots \in R[[x ; \sigma]]$. Since $a b c \neq 0, R$ is not $\sigma$-skew power-serieswise Armendariz. Note that if $(a-a b x) d=0$ for some $d \in R$, then $a d=0$ and $a b d=0$, and so $d=0$.

Cortes in [8, Proposition 3.2] (resp. [9, Lemma 2.7]) and observed the relations between annihilators in a ring $R$ and annihilators in $R[x ; \sigma]$ (resp. $R[[x ; \sigma]])$ in case of $\sigma$ being an automorphism of $R$. We here extend the Cortes' results to the situation of $\sigma$-skew power series ring in case of $\sigma$ being an endomorphism. This result provides useful tools for studying the skew power series rings with the $\sigma$-skew power-serieswise Armendariz condition, and its applications. For a ring $R$, let $f(x)=\sum_{i=0}^{\infty} a_{i} x^{i} \in R[[x ; \sigma]]$ and $A_{f}=\left\{a_{i} x^{i} \mid i \in I\right\}$, where $I$ is the set of all nonnegative integers. Then for each $i, r_{R}\left(a_{i} x^{i}\right)=$ $r_{R[[x ; \sigma]]}\left(a_{i} x^{i}\right) \cap R$ is a right ideal of $R$ and $r_{R[[x ; \sigma]]}\left(A_{f}\right)=\cap_{i} r_{R[[x ; \sigma]]}\left(a_{i} x^{i}\right)$; hence $r_{R}\left(A_{f}\right)=\left(\cap_{i} r_{R[[x ; \sigma]]}\left(a_{i} x^{i}\right)\right) \cap R$ is a right ideal of $R$. Let

$$
\begin{aligned}
& \Gamma=\left\{r_{R}\left(A_{f}\right) \mid f(x)=\sum_{i=0}^{\infty} a_{i} x^{i} \in R[[x ; \sigma]]\right\}, \\
& \Delta=\left\{r_{R[[x ; \sigma]]}(V) \mid \emptyset \neq V \subseteq R[[x ; \sigma]]\right\} .
\end{aligned}
$$

Then we obtain a map $\Phi: \Gamma \rightarrow \Delta$ defined by $\Phi\left(r_{R}\left(A_{f}\right)\right)=r_{R}\left(A_{f}\right) R[[x ; \sigma]]$ as in the proof of Lemma 1.8 to follow.

Lemma 1.8. Let $\sigma$ be an endomorphism of a ring $R$. Then the following statements are equivalent:

(1) $R$ is $\sigma$-skew power-serieswise Armendariz;

(2) $\Phi: \Gamma \rightarrow \Delta$ is bijective with $\Phi\left(r_{R}\left(A_{f}\right)\right)=r_{R}\left(A_{f}\right) R[[x ; \sigma]]$.

Proof. (1) $\Rightarrow(2)$ : We first claim that $\Phi$ is well-defined. Let $f(x)=\sum_{i=0}^{\infty} a_{i} x^{i} \in$ $R[[x ; \sigma]]$ and $g(x)=\sum_{i=0}^{\infty} b_{i} x^{i} \in r_{R}\left(A_{f}\right) R[[x ; \sigma]]$ for $r_{R}\left(A_{f}\right) \in \Gamma$. Then we can assume that $b_{i} \in r_{R}\left(A_{f}\right)$ for all $i$ and we have that $b_{\ell} x^{\ell} \in r_{R[[x ; \sigma]]}\left(A_{f}\right)$ for each $\ell$. Thus, $g(x) \in r_{R[[x ; \sigma]]}\left(A_{f}\right)$. On the other hand, let $h(x)=\sum_{i=0}^{\infty} c_{i} x^{i} \in$ $r_{R[[x ; \sigma]]}\left(A_{f}\right)$. Then $0=a_{i} x^{i}\left(\sum_{i=0}^{\infty} c_{i} x^{i}\right)=a_{i} x^{i} c_{0}+a_{i} x^{i} c_{1} x+\cdots+a_{i} x^{i} c_{n} x^{n}+\cdots$ for all $i$. If $a_{i} x^{i} c_{t} \neq 0$ for some $t$, then $a_{i} \sigma^{i}\left(c_{t}\right) \neq 0$ and it follows that $a_{i} x^{i} c_{t} x^{t}=$ $a_{i} \sigma^{i}\left(c_{t}\right) x^{i+t} \neq 0$. Hence $a_{i} x^{i} h(x) \neq 0$, a contradiction. Thus $c_{k} \in r_{R}\left(A_{f}\right)$ for all $k$, and we get $h(x) \in r_{R}\left(A_{f}\right) R[[x ; \sigma]]$. Consequently $r_{R}\left(A_{f}\right) R[[x ; \sigma]]=$ $r_{R[[x ; \sigma]]}\left(A_{f}\right) \in \Delta$, which determines the map $\Phi: \Gamma \rightarrow \Delta$ with $\Phi\left(r_{R}\left(A_{f}\right)\right)=$ $r_{R}\left(A_{f}\right) R[[x ; \sigma]]$. We next show that $\Phi$ is injective. In fact, $\Phi\left(r_{R}\left(A_{f}\right)\right)=$ $\Phi\left(r_{R}\left(A_{g}\right)\right)$ for $f(x), g(x) \in R[[x ; \sigma]]$. Then $r_{R}\left(A_{f}\right) R[[x ; \sigma]]=r_{R}\left(A_{g}\right) R[[x ; \sigma]]$ and we have that $r_{R[[x ; \sigma]]}\left(A_{f}\right)=r_{R[[x ; \sigma]]}\left(A_{g}\right)$ what we proved above. It then follows

$$
r_{R}\left(A_{f}\right)=r_{R[[x ; \sigma]]}\left(A_{f}\right) \cap R=r_{R[[x ; \sigma]]}\left(A_{g}\right) \cap R=r_{R}\left(A_{g}\right),
$$

which implies that $\Phi$ is injective. 
We claim that $\Phi$ is surjective. In fact, let $V$ be a subset of $R[[x ; \sigma]]$ and $f(x)=\sum_{i=0}^{\infty} a_{i} x^{i} \in V$. If $g(x)=\sum_{i=0}^{\infty} b_{i} x^{i} \in r_{R[[x ; \sigma]]}(f(x))$, then $f(x) g(x)=$ 0 . Since $R$ is $\sigma$-skew power-serieswise Armendariz, we have $a_{i} \sigma^{i}\left(b_{j}\right)=0$ for each $i, j$. Thus for any $j$, we have $b_{j} \in \cap_{i} r_{R}\left(a_{i} x^{i}\right)$, and it follows that $g(x) \in r_{R}\left(A_{f}\right) R[[x ; \sigma]]$. Hence $r_{R[[x ; \sigma]]}(f(x)) \subseteq r_{R}\left(A_{f}\right) R[[x ; \sigma]]$. On the other hand, let $g(x) \in r_{R}\left(A_{f}\right) R[[x ; \sigma]]=r_{R[[x ; \sigma]]}\left(A_{f}\right)$. Then we get $f(x) g(x)=0$ which implies that $g(x) \in r_{R[[x ; \sigma]]}(f(x))$, and we have that $r_{R[[x ; \sigma]]}(f(x))=$ $r_{R}\left(A_{f}\right) R[[x ; \sigma]]=r_{R[[x ; \sigma]]}\left(A_{f}\right)$. Consequently

$$
\begin{aligned}
r_{R[[x ; \sigma]]}(V) & =\bigcap_{f(x) \in V} r_{R[[x ; \sigma]]}(f(x))=\bigcap_{f(x) \in V} r_{R[[x ; \sigma]]}\left(A_{f}\right)=r_{R[[x ; \sigma]]}\left(\bigcup_{f(x) \in V} A_{f}\right) \\
& =r_{R[[x ; \sigma]]}\left(A_{V}\right)=r_{R}\left(A_{V}\right) R[[x ; \sigma]]=\Phi\left(r_{R}\left(A_{V}\right),\right.
\end{aligned}
$$

where $A_{V}=\cup_{f(x) \in V} A_{f}$, completing the proof.

$(2) \Rightarrow(1)$ : Let $f(x)=\sum_{i=0}^{\infty} a_{i} x^{i}, g(x)=\sum_{i=0}^{\infty} b_{i} x^{i} \in R[[x ; \sigma]]$ with $f(x) g(x)$ $=0$. Since $\Phi$ is surjective, $g(x) \in r_{R[[x ; \sigma]]}(f(x))=r_{R}\left(A_{h}\right) R[[x ; \sigma]]$ for some $h(x)=\sum_{i=0}^{\infty} c_{i} x^{i} \in R[[x ; \sigma]]$. Thus $b_{k} \in r_{R}\left(A_{h}\right) \subseteq r_{R[[x ; \sigma]]}(f(x))$, and hence $f(x) b_{k}=0$ for all $k$. This implies $a_{i} \sigma^{i}\left(b_{k}\right)=0$ for all $i, k$. So $R$ is $\sigma$-skew power-serieswise Armendariz.

Cortes [8, Corollary 3.10] proved that a $\sigma$-skew Armendariz ring $R$ with an automorphism $\sigma$ is right Goldie if and only if $R[x ; \sigma]$ is right Goldie. But, there exists a $\sigma$-skew power-serieswise Armendariz $\operatorname{ring} R$ with finite right Goldie dimension whose skew power series ring $R[[x ; \sigma]]$ has no a finite right Goldie dimension [18, Example 10.31A and Theorem 10.22].

Proposition 1.9. Let $R$ be a $\sigma$-skew power-serieswise Armendariz ring with an endomorphism $\sigma$. Then $R$ satisfies the ascending chain condition on right annihilators if and only if $R[[x ; \sigma]]$ satisfies the ascending chain condition on right annihilators.

Proof. It suffices to show the necessity. Suppose that $r_{R[[x ; \sigma]]}\left(V_{1}\right) \subseteq r_{R[[x ; \sigma]]}\left(V_{2}\right)$ $\subseteq \cdots$ is an ascending chain in $R[[x ; \sigma]]$ for $V_{i} \subseteq R[[x ; \sigma]]$. By Lemma 1.8, $r_{R[[x ; \sigma]]}\left(V_{i}\right)=r_{R}\left(A_{V_{i}}\right) R[[x ; \sigma]]$, where $A_{V}=\cup_{f(x) \in V} A_{f}$ and we have that $r_{R}\left(A_{V_{i}}\right) \subseteq r_{R}\left(A_{V_{i+1}}\right)$. Let $a \in r_{R}\left(A_{V_{i}}\right)$. Then $A_{V_{i}} a=0$ and we have that $f(x) a=0$ for any $f(x) \in V_{i}$. Thus $V_{i+1} a=0$ and it follows that $A_{V_{i+1}} a=0$. Hence $r_{R}\left(A_{V_{i}}\right) \subseteq r_{R}\left(A_{V_{i+1}}\right)$. By assumption, there exists an integer $n \geq 1$ such that $r_{R}\left(A_{V_{n}}\right)=r_{R}\left(A_{V_{n+k}}\right)$ for all integers $k \geq 1$. So $r_{R[[x ; \sigma]]}\left(V_{n}\right)=$ $r_{R[[x ; \sigma]]}\left(V_{n+k}\right)$.

For a ring $R$, let $I$ be an ideal and $\sigma$ be an automorphism of $R$. If $\sigma(I) \subseteq I$, then $I$ is called a $\sigma$-ideal. If $\sigma(I)=I$, then $I$ is called $\sigma$-invariant. According to Pearson and Stephenson [24], a proper $\sigma$-ideal $P$ of $R$ is $\sigma$-prime if whenever $A B \subseteq P$ for an ideal $A$ and a $\sigma$-ideal $B$, we have that either $A \subseteq P$ or $B \subseteq \bar{P}$. If in addition $P$ is $\sigma$-invariant, then $P$ is called strongly $\sigma$-prime. We mean by a right annihilator ideal (left annihilator ideal), an ideal of the form 
$r_{R}(I)$, (respectively, $\ell_{R}(I)$ ), where $I$ is an ideal of $R$. If an ideal is of the form $r_{R}(I)=\ell_{R}(I)$, where $I$ is a $\sigma$-ideal of $R$, then it will be called an annihilator $\sigma$-ideal. By an annihilator $\sigma$-prime ideal, we mean an annihilator $\sigma$-ideal which is also $\sigma$-prime.

The following lemma is due to [13, Lemma 1.2].

Lemma 1.10. Let $P$ be a proper $\sigma$-invariant ideal of a ring $R$ with an automorphism $\sigma$. Then the following conditions are equivalent:

(1) $P$ is strongly $\sigma$-prime;

(2) For $a, b \in R$, if for some positive integer $m$, aR $\sigma^{n}(b) \subseteq P$ for all $n \geq m$, then $a \in P$ or $b \in P$;

(3) For $a, b \in R$, if for some integer $m, a R \sigma^{n}(b) \subseteq P$ for all $n \geq m$, then $a \in P$ or $b \in P$.

A strongly $\sigma$-prime ideal is obviously $\sigma$-prime, but otherwise "strongly $\sigma$ prime", " $\sigma$-prime" and "prime" are completely independent conditions (see, $[20])$.

By [23, Proposition 1.6] and [24, Corollary 1.4], we have the following.

Proposition 1.11. Let $\sigma$ be an automorphism of $R$. If $R$ is a semiprime ring with the ascending chain condition on right annihilators, then we have

(1) $R$ has only a finite number of minimal strongly $\sigma$-prime ideals, and their intersection is zero.

(2) A strongly $\sigma$-prime ideal of $R$ is minimal if and only if it is an annihilator $\sigma$-ideal.

(3) $R[[x ; \sigma]]$ is semiprime.

Even though, if $R$ is a semiprime ring with the ascending chain condition on right annihilators, a minimal strongly $\sigma$-prime ideal is not necessarily to be a minimal prime ideal. For example, let $R=\mathbb{Z}_{2} \oplus \mathbb{Z}_{2}$ and let $\sigma: R \rightarrow R$ be an automorphism defined by $\alpha((a, b))=(b, a)$. Then the minimal (strongly) $\sigma$-prime ideal $\{(0,0)\}$ of the ring $R$ is not minimal prime.

Theorem 1.12. Let $R$ be a semiprime ring with the ascending chain condition on right annihilators. If $R$ is a $\sigma$-skew power-serieswise Armendariz ring with an automorphism $\sigma$, then $R[[x ; \sigma]]$ has finitely many minimal strongly $\bar{\sigma}$-prime ideals, say $Q_{1}, \ldots, Q_{m}$, such that $Q_{1} \cap \cdots \cap Q_{m}=0$ and $Q_{i}=P_{\tau(i)}[[x ; \sigma]]$ for any $i$, where $P_{\tau(1)}, \ldots, P_{\tau(m)}$ are minimal strongly $\sigma$-prime ideals of $R$ and $\tau$ is a permutation of $\{1, \ldots, m\}$.

Proof. By Propositions 1.11 and $1.9, R[[x ; \sigma]]$ is a semiprime ring with the ascending chain condition on right annihilators. Thus $R[[x ; \sigma]]$ has finitely many minimal strongly $\bar{\sigma}$-prime ideals, say $Q_{1}, \ldots, Q_{m}$. By Proposition 1.11(2), $Q_{i}=r_{R[[x ; \bar{\sigma}]]}\left(I_{i}\right)$ for any $i$, where $I_{i}$ is an ideal of $R[[x ; \sigma]]$. Then by Lemma 1.8, $Q_{i}=r_{R}\left(A_{I_{i}}\right) R[[x ; \sigma]]$, where $A_{I_{i}}=\cup_{f(x) \in I_{i}}\left(A_{f}\right)$.

We now note that $R$ is $\sigma$-rigid (so $R[[x ; \sigma]]$ is $\bar{\sigma}$-rigid). Suppose $a \sigma(a)=0$ for $a \in R$. By Lemma 1.4(2), $a R \sigma^{2}(a)=0$. Since $R$ is semiprime, $\sigma^{2}(a) R a=0$ 
and so $\sigma^{2}(a) a=0$. Again applying to Lemma $1.4(2), \sigma^{2}(a) R \sigma^{2}(a)=0$. Since $\sigma$ is an automorphism, we have $a R a=0$, which yields $a=0$.

We claim that $C$ is a strongly $\sigma$-prime ideal of $R$ if and only if $C[[x ; \sigma]]$ is strongly $\bar{\sigma}$-prime of $R[[x ; \sigma]]$. Suppose that $C[[x ; \sigma]]$ is strongly $\bar{\sigma}$-prime of $R[[x ; \sigma]]$. Note that $\sigma(C)[[x ; \sigma]]=\bar{\sigma}(C[[x ; \sigma]])=C[[x ; \sigma]]$, and we have that $\sigma(C)=C$. Suppose that for some integer $m, a R \sigma^{n}(b) \subseteq C$ for all $n \geq m$ with $b \notin C$. As mentioned above, $C=r_{R}(I)$ for some ideal $I$ of $R$. Then $y \notin C$ for all $y \in I$ since $R$ is reduced. Thus $y a \sigma^{n}(b)=0$ and $y R[[x ; \sigma]] \bar{\sigma}^{s}(b) \nsubseteq$ $C[[x ; \sigma]]$ for some integer $s$ by Lemma 1.10. This implies that $y r \sigma^{t}(b) \notin C$ for some integer $t$ and $r \in R$. Since $y a b=0$ and $R[[x ; \sigma]]$ is $\bar{\sigma}$-rigid, then $y r \sigma^{m}(b) R[[x ; \sigma]] \sigma^{l}(a)=0 \subseteq C[[x ; \sigma]]$ for any integer $l$, and it follows that $a \in C$ which implies that $C$ is strongly $\sigma$-prime.

Conversely, we suppose that $f(x)=\sum_{i=0}^{\infty} a_{i} x^{i}, g(x)=\sum_{j=0}^{\infty} b_{j} x^{j}$ are in $R[[x ; \sigma]]$ such that $f(x) R[[x ; \sigma]] \bar{\sigma}^{m}(g(x)) \subseteq C[[x ; \sigma]]$ for all integer $n \geq m$, where $m$ is an integer, and $g(x) \notin C[[x ; \sigma]]$. Then there exists $b_{t}$ which is minimal among such that $b_{i} \notin C$, where $0 \leq t$. Then for any integer $s \geq 1$ and $t>j, \sigma^{s}\left(b_{j}\right) \in C$ since $\sigma(C)=C$. Note that

$$
a_{0} R \sigma^{m}\left(b_{t}\right)+a_{1} R \sigma^{m+1}\left(b_{t-1}\right)+\cdots+a_{t} R \sigma^{m+t}\left(b_{0}\right) \subseteq C .
$$

Then $a_{0} R \sigma^{m}\left(b_{t}\right) \subseteq C$ and we have that $a_{0} \in C$. Also since $a_{0} R \sigma^{m}\left(b_{t+1}\right)+$ $a_{1} R \sigma^{m+1}\left(b_{t}\right)+\cdots+a_{t+1} R \sigma^{m+t}\left(b_{0}\right) \subseteq C, a_{1} R \sigma^{m}\left(b_{t}\right) \subseteq C$ and it follows that $a_{1} \in C$. Repeating this process, we have $f(x) \in C[[x ; \sigma]]$, which implies that $C[[x ; \sigma]]$ is strongly $\bar{\sigma}$-prime in $R[[x ; \sigma]]$. Moreover, $C$ is a minimal strongly $\sigma$ prime ideal of $R$ if and only if $C[[x ; \sigma]]$ is minimal strongly $\bar{\sigma}$-prime in $R[[x ; \sigma]]$.

Using the fact, we have for any $i, P_{i}=r_{R}\left(A_{I_{i}}\right)$ is minimal strongly $\sigma$-prime in $R$, we get $t=m$ and we obtain that $Q_{i}=P_{i}[[x ; \sigma]]$ for all $i=1, \ldots, m$ (after reordering if necessary).

\section{Skew IFP rings}

In this section, we study some properties of $\sigma$-skew IFP rings. According to Baser et al. [3, Definition 2.1], for an endomorphism $\sigma$ of a ring $R, R$ is called $\sigma$-skew IFP if for $a, b \in R, a b=0$ implies $a R \sigma(b)=0$. In [3, Theorem 2.4] they proved that a ring $R$ is $\sigma$-rigid if and only if $R$ is reduced $\sigma$-skew IFP and $\sigma$ is a monomorphism. An endomorphism $\sigma$ of $R$ is of locally finite order if for every $r \in R$ there exists a positive integer $n=n(r)$ such that $\sigma^{n}(r)=r$.

We begin with the following lemma.

Lemma 2.1. For a ring $R$ with an endomorphism $\sigma$, we have the following:

(1) $A$ ring $R$ is $\sigma$-skew IFP if and only if for $a, b \in R, a b=0$ implies $a R \sigma^{n}(b)=0$ for all integers $n \geq 1$

(2) A $\sigma$-skew power-serieswise Armendariz ring is $\sigma$-skew IFP;

(3) If $\sigma$ is of locally finite order, then a $\sigma$-skew IFP ring is IFP. 
Proof. (1) Let $R$ be a $\sigma$-skew IFP ring and $a b=0$ for $a, b \in R$. Then $a R \sigma(b)=$ 0 and in particular $a \sigma(b)=0$. Since $R$ is $\sigma$-skew IFP, we have $a R \sigma^{2}(b)=0$. Inductively we obtain the result.

(2) It follows from (1) and Lemma 1.4(2).

(3) Let $\sigma$ be an endomorphism of a ring $R$ with $\sigma^{n}(b)=b$ and $a b=0$ for $a, b \in R$. Then we get $a R b=a R \sigma^{n}(b)=0$ by (2), and so $R$ is IFP.

Baser et al. [3, Theorem 3.3(3)] proved that $R[[x ; \sigma]]$ is IFP when $R$ is a $\sigma$-sps Armendariz $\sigma$-IFP ring. However, by Lemma 2.1(2), we can remove the condition " $R$ is $\sigma$-IFP" in the result.

Note that a $\sigma$-skew IFP ring and an IFP ring coincide when $\sigma$ is an identity map. The converses of Lemma 2.1(1) and (3) does not need to hold as we see in the following example.

Example 2.2. (1) Let $D$ be a commutative domain. Suppose that $X=$ $\left\{t_{j} \mid j \in \mathbb{Z}\right\}$ is a set of commuting indeterminates over $D$ and $F$ is the quotient field of $D[X]$ and $\alpha$ an automorphism of $F$ defined by $t_{i} \mapsto t_{i+1}$. The construction of the following ring $R$ is due to Hamann and Swan [11].

Let $S=F[A]$ be the free algebra generated by the noncommuting indeterminates $A=\left\{a_{i} \mid i=0,1,2, \ldots\right\}$ over $F, I$ the ideal of $S$ generated by $\left\{a_{i} a_{j} a_{k} \mid a_{i}, a_{j}\right.$ and $a_{k}$ are in $\left.A\right\}, T=\frac{S}{I}$ and identify $a_{i}$ with $a_{i}+I$ for simplicity. Next we let $d_{n}=\sum_{i+j=n} a_{i} a_{j}$ and $J$ the ideal of $T$ generated by $\left\{d_{n} \mid n=0,1,2, \ldots\right\}$. Now we put $R=\frac{T}{J}$, and define an automorphism $\sigma$ of $R$ by $\sigma\left(\sum_{\text {finite }} s Y\right) \rightarrow \sum_{\text {finite }} \alpha(s) Y$ where $s \in F$ and $Y$ is a finite product of $a_{i}$ 's.

Consider $f(x)=\sum_{i=0}^{\infty} a_{i} x^{i} \in R[[x ; \sigma]]$. Note that $f(x)^{2}=0$, however $a_{j} \sigma^{j}\left(a_{k}\right)=a_{j} a_{k} \neq 0$ unless $j, k$ are zero. Thus $R$ is not $\sigma$-skew power-serieswise Armendariz.

We next show that $R$ is $\sigma$-skew IFP. In fact, let $L_{1}$ and $L_{2}$ be the linear spaces generated by $\left\{a_{n} \mid a_{n} \in A\right\}$ and $\left\{a_{i} a_{j} \mid a_{i}\right.$ and $a_{j}$ are in $\left.A\right\}$ over $F$, respectively, and $b, c \in S$ such that $b c \in J$. Note that $b, c$ can be expressed by

$$
b=b_{0}+b_{1}+b_{2}+b_{3} \text { and } c=c_{0}+c_{1}+c_{2}+b_{3}
$$

for some $b_{0}, c_{0} \in F, b_{1}, c_{1} \in L_{1}, b_{2}, c_{2} \in L_{2}$, and $b_{3}, c_{3} \in I$. From $b c \in J$ we get $b_{0} c_{0}=0$, and it follows that $b_{0}=0$ or $c_{0}=0$. Suppose that $b_{0}=0$ and $c_{0} \neq 0$. Then we get

$$
c_{0} b_{1}+\left(c_{0} b_{2}+b_{1} c_{1}\right) \in J \text { with } c_{0} b_{1} \in L_{1}, c_{0} b_{2}+b_{1} c_{1} \in L_{2},
$$

and we have that $c_{0} b_{1}=0$ and $c_{0} b_{2}+b_{1} c_{1} \in J$. From $c_{0} \neq 0$ we have $b_{1}=$ 0 ; hence $c_{0} b_{2} \in J$ and we have that $c_{0} b_{2}=0$, which implies that $b_{2}=0$. Consequently $b=b_{3} \in I \subseteq J$ and so $b R \sigma(c)=0$. Next suppose that $b_{0}=0=$ $c_{0}$. Then we get $b_{1} c_{1} \in J$ with $b_{1} c_{1} \in L_{2}$. We can write $b_{1}=\sum_{s=0}^{m} v_{s} a_{i_{s}}$ and $c_{1}=\sum_{t=0}^{n} w_{t} a_{j_{t}}$, where $v_{s}, w_{t} \in F$ and $a_{i_{s}}, a_{j_{t}} \in A$. Thus

$$
b_{1} c_{1}=\left(\sum_{s=0}^{m} v_{s} a_{i_{s}}\right)\left(\sum_{t=0}^{n} w_{t} a_{j_{t}}\right)=\sum_{s, t} v_{s} w_{t} a_{i_{s}} a_{j_{t}} \in J \cap L_{2} .
$$


In this situation we can assume that $a_{i_{\alpha}} \neq a_{i_{\beta}}$ if $i_{\alpha} \neq i_{\beta}$ and $a_{j_{\delta}} \neq a_{j_{\gamma}}$ if $j_{\delta} \neq j_{\gamma}$. So there exist $a_{i_{\delta}}$ and $a_{j_{\gamma}}$ such that $i_{\delta}$ and $j_{\gamma}$ are largest in the indices $i_{s}$ 's and $j_{t}$ 's respectively.

Since $i_{\delta}+j_{\gamma}$ is larger than every other $i_{s}+j_{t}$, we have $a_{i_{\delta}} a_{j_{\gamma}}$ must be $d_{0}$ (i.e., $a_{i_{\delta}}=a_{0}=a_{j_{\gamma}}$ ). Hence we have that

$$
b_{1}=v a_{0} \text { and } c_{1}=w a_{0}
$$

for some $v, w \in F$ since $i_{\delta}$ and $j_{\gamma}$ are largest. So we get

$$
\begin{aligned}
b R \sigma(c) & =\left(v a_{0}+b_{2}\right) R \sigma\left(w a_{0}+c_{2}\right) \\
& =\left(v a_{0}+b_{2}\right)\left(e_{0}+e_{1}+e_{2}\right)\left(\alpha(w) a_{0}+\sigma\left(c_{2}\right)\right) \\
& =v e_{0} \alpha(w) a_{0}^{2}=0
\end{aligned}
$$

for all $e_{0} \in F, e_{1} \in L_{1}, e_{2} \in L_{2}$.

The computation for the case of $c=0$ is similar, and therefore $R$ is $\sigma$-skew IFP.

(2) There is an IFP ring that is not $\sigma$-skew IFP with $\sigma$ of infinite order. Let $S$ be a domain and $R=\sum_{i \in \mathbb{Z}} S_{i}$ with $S_{i}=S$ for all $\in \mathbb{Z}$. Then $R$ is IFP since $R$ is reduced. Define $\sigma\left(a_{i}\right)=\left(a_{i+1}\right)$, then $\sigma$ is an automorphism of $R$ of infinite order. Take $\left(a_{i}\right)_{i \in \mathbb{Z}},\left(b_{j}\right)_{j \in \mathbb{Z}} \in R$ such that $a_{i}=0=b_{j}$ for all odd integers $i$, even integers $j$, and $a_{i}=1=b_{j}$ for all even integers $i$, odd integers $j$. Then $\left(a_{i}\right)_{i \in \mathbb{Z}}\left(b_{j}\right)_{j \in \mathbb{Z}}=0$, but $\left(a_{i}\right)_{i \in \mathbb{Z}} R \sigma\left(b_{j}\right)_{j \in \mathbb{Z}}=\left(a_{i}\right)_{i \in \mathbb{Z}} R\left(a_{i}\right)_{i \in \mathbb{Z}} \neq 0$. Thus $R$ is not $\sigma$-skew IFP.

Note that if the skew power series ring $R[[x ; \sigma]]$ is IFP, then $R$ is IFP and $\sigma$-skew IFP. Conversely, if $R$ is a $\sigma$-skew IFP ring with of locally finite order and $\sigma$-skew power-serieswise Armendariz ring, then $R[[x ; \sigma]]$ is IFP. However, the following example shows that the condition " $R$ is $\sigma$-skew power-serieswise Armendariz" is essential.

Example 2.3. We modify the construction and apply the computation in $[15$, Example]. Let $\mathbb{Z}_{2}$ be the field of integers modulo 2 and $A=\mathbb{Z}_{2}\left\{a_{0}, a_{1}, a_{2}, b_{0}, b_{1}\right.$, $\left.b_{2}, c\right\}$ be the free algebra of polynomials with zero constant terms in noncommuting indeterminates $a_{0}, a_{1}, a_{2}, b_{0}, b_{1}, b_{2}, c$ over $\mathbb{Z}_{2}$. Set $S=\mathbb{Z}_{2}+A$ and consider an ideal of $S$, say $I$, generated by

$$
\begin{gathered}
a_{0} b_{0}, a_{1} b_{2}+a_{2} b_{1}, a_{0} b_{1}+a_{1} b_{0}, a_{0} b_{2}+a_{1} b_{1}+a_{2} b_{0}, a_{2} b_{2}, a_{0} r b_{0}, a_{2} r b_{2}, \\
\left(a_{0}+a_{1}+a_{2}\right) r\left(b_{0}+b_{1}+b_{2}\right), \\
a_{0} a_{0}, a_{1} a_{2}+a_{2} a_{1}, a_{0} a_{1}+a_{1} a_{0}, a_{0} a_{2}+a_{1} a_{1}+a_{2} a_{0}, a_{2} a_{2}, a_{0} r a_{0}, a_{2} r a_{2}, \\
\left(a_{0}+a_{1}+a_{2}\right) r\left(a_{0}+a_{1}+a_{2}\right), \\
a_{1} a_{2}+a_{2} b_{1}, a_{0} b_{1}+a_{1} a_{0}, a_{0} b_{2}+a_{1} a_{1}+a_{2} b_{0}, \\
b_{0} a_{0}, b_{1} a_{2}+b_{2} a_{1}, b_{0} a_{1}+b_{1} a_{0}, b_{0} a_{2}+b_{1} a_{1}+b_{2} a_{0}, b_{2} a_{2}, b_{0} r a_{0}, b_{2} r a_{2}, \\
\left(b_{0}+b_{1}+b_{2}\right) r\left(a_{0}+a_{1}+a_{2}\right), \\
b_{0} b_{0}, b_{1} b_{2}+b_{2} b_{1}, b_{0} b_{1}+b_{1} b_{0}, b_{0} b_{2}+b_{1} b_{1}+b_{2} b_{0}, b_{2} b_{2}, b_{0} r b_{0}, b_{2} r b_{2},
\end{gathered}
$$




$$
\begin{gathered}
\left(b_{0}+b_{1}+b_{2}\right) r\left(b_{0}+b_{1}+b_{2}\right), \\
b_{1} b_{2}+b_{2} a_{1}, b_{0} a_{1}+b_{1} b_{0}, b_{0} a_{2}+b_{1} b_{1}+b_{2} a_{0}
\end{gathered}
$$

with $r \in A$ and $r_{1} r_{2} r_{3} r_{4}$ with $r_{1}, r_{2}, r_{3}, r_{4} \in A$. Then clearly $A^{4} \in I$. Now let $R=\left(\mathbb{Z}_{2}+A\right) / I$ and identify every element in $S$ with its image in $R$ for simplicity. Define a map $\sigma$ from $R$ to $R$ by $\sigma\left(a_{i}\right)=b_{i}, \sigma\left(b_{i}\right)=a_{i}$, and $\sigma(c)=c$ for $i=0,1,2$. Note that $\sigma$ is an automorphism of $R$ (also of $S$ ) with $\sigma(I)=I$.

We consider $f(x)=a_{0}+a_{1} x+a_{2} x^{2}, g(x)=b_{0}+b_{1} x+b_{2} x^{2} \in R[x ; \sigma]$, such that $f(x) g(x)=0$. Thus $\left(a_{0}+a_{1} x+a_{2} x^{2}\right) c\left(b_{0}+b_{1} x+b_{2} x^{2}\right) \neq 0$ because $a_{0} c b_{1}+a_{1} c a_{0} \neq 0$. Hence $R[x ; \sigma]$ (and so $R[[x ; \sigma]]$ ) is not IFP.

We next show that $R$ is $\sigma$-skew IFP. In fact, each product of indeterminates $a_{0}, a_{1}, a_{2}, b_{0}, b_{1}, b_{2}, c$ is called a monomial and we say that $\alpha$ is a monomial of degree $n$ if it is a product of exactly $n$ generators. Let $H_{n}$ be the set of all linear combinations of monomials of degree $n$ over $\mathbb{Z}_{2}$. Observe that $H_{n}$ is finite for any $n$ and that the ideal $I$ of $R$ is homogeneous (i.e., if $\sum_{i=1}^{s} r_{i} \in I$ with $r_{i} \in H_{i}$, then every $r_{i}$ is in $I$ ). Note that $\sigma^{2}$ is the identity map of $R$.

Claim 1. If $f_{1} g_{1} \in I$ with $f_{1}, g_{1} \in H_{1}$, then $f_{1} r \sigma^{k}\left(g_{1}\right) \in I$ for any $r \in S$ and $k \geq 1$.

Proof. Let $f_{1} g_{1} \in I$ with $f_{1}, g_{1} \in H_{1}$. Then, by the definition of $I$, we obtain the following cases: $\left(f_{1}=a_{0}, g_{1}=b_{0}\right),\left(f_{1}=a_{2}, g_{1}=b_{2}\right),\left(f_{1}=a_{0}+a_{1}+a_{2}, g_{1}=\right.$ $\left.b_{0}+b_{1}+b_{2}\right),\left(f_{1}=a_{0}, g_{1}=a_{0}\right),\left(f_{1}=a_{2}, g_{1}=a_{2}\right),\left(f_{1}=a_{0}+a_{1}+a_{2}, g_{1}=\right.$ $\left.a_{0}+a_{1}+a_{2}\right),\left(f_{1}=b_{0}, g_{1}=a_{0}\right),\left(f_{1}=b_{2}, g_{1}=a_{2}\right),\left(f_{1}=b_{0}+b_{1}+b_{2}, g_{1}=\right.$ $\left.a_{0}+a_{1}+a_{2}\right),\left(f_{1}=b_{0}, g_{1}=b_{0}\right),\left(f_{1}=b_{2}, g_{1}=b_{2}\right)$, or $\left(f_{1}=b_{0}+b_{1}+b_{2}, g_{1}=\right.$ $\left.b_{0}+b_{1}+b_{2}\right)$. So we complete the proof, using the definition of $I$ again.

Claim 2. If $f g \in I$ with $f, g \in A$, then $f r \sigma^{k}(g) \in I$ for all $r \in A$.

Proof. Observe that $f=f_{1}+f_{2}+f_{3}+f_{4}, g=g_{1}+g_{2}+g_{3}+g_{4}$ and $r=$ $r_{1}+r_{2}+r_{3}+r_{4}$ for some $f_{1}, g_{1}, r_{1} \in H_{1}, f_{2}, g_{2}, r_{2} \in H_{2}, f_{3}, g_{3}, r_{3} \in H_{3}$ and some $f_{4}, g_{4}, r_{4} \in I$. Note that $H_{i} \subseteq I$ for $i \geq 4$. So $f r \sigma^{k}(g)=f_{1} r_{1} \sigma^{k}\left(g_{1}\right)+h$ for some $h \in I$. Since $f g \in I$ then $f_{1} g_{1} \in I$ by the fact that $I$ is homogeneous; we have that $f_{1} r_{1} \sigma^{k}\left(g_{1}\right) \in I$ by Claim 1 . This gives us $f r \sigma^{k}(g) \in I$.

We claim that $R$ is $\sigma$-skew IFP. In fact, it suffices to show that $y r \sigma^{k}(z) \in I$ for all $r \in S$ whenever $y z \in I$ for $y, z \in S$. By Claim 2, we can obtain that $y=\alpha+y^{\prime}, z=\beta+z^{\prime}$ for some $\alpha, \beta \in \mathbb{Z}_{2}$ and some $y^{\prime}, z^{\prime} \in A$. Hence, $\alpha \beta+\alpha z^{\prime}+y^{\prime} \beta+y^{\prime} z^{\prime}=y z \in I$ and it follows that $\alpha=0$ or $\beta=0$. Assume that $\alpha=0$. Then $y^{\prime} \beta+y^{\prime} z^{\prime} \in I$. If $\beta \neq 0$, then $y^{\prime} \in I$ because $I$ is homogeneous, $\beta \in \mathbb{Z}_{2}$ and consequently $y r \sigma^{k}(z)=y^{\prime} r \sigma^{k}(z) \in I$ for all $r \in \mathbb{Z}_{2}+A$. If $\beta=0$, then $y^{\prime} z^{\prime} \in I$ and so by Claim 2, yr $\sigma^{k}(z)=y^{\prime} r \sigma^{k}\left(z^{\prime}\right) \in I$ for all $r \in \mathbb{Z}_{2}+A$. The proof of the case of $\beta=0$ is similar. Therefore $R$ is a $\sigma$-skew IFP ring.

By [16, Lemma 2.3], if a ring $R$ is power-serieswise Armendariz, then $R[[x]]$ is IFP. However, we now do not know that $R[[x ; \sigma]]$ is IFP when a ring $R$ is $\sigma$-skew power-serieswise Armendariz. Note that, in Example 2.3, the ring $R$ is a $\sigma$-skew IFP ring with $\sigma$ of locally finite order, but $R[[x ; \sigma]]$ is not IFP. 
Proposition 2.4. If $R$ is a $\sigma$-skew power-serieswise Armendariz ring with $\sigma$ of locally finite order, then $R[[x ; \sigma]]$ is IFP, where $\sigma$ is an endomorphism of $R$.

Proof. Let $R$ be a $\sigma$-skew power-serieswise Armendariz ring with $\sigma$ of locally finite order. Suppose $f(x) g(x)=0$ for $f(x)=\sum_{i=0}^{\infty} a_{i} x^{i}, g(x)=\sum_{j=0}^{\infty} b_{j} x^{j} \in$ $R[[x ; \sigma]]$. Then $a_{i} \sigma^{i}\left(b_{j}\right)=0$ for all $i, j$. By Lemma 2.1(2) and (3), we have $a_{i} R \sigma^{i+k}\left(b_{j}\right)=0$ for all $i, j$ where $k \geq 0$. This yields $f(x) R x^{k} g(x)=0$ for all $k \geq 0$ and so $f(x) R[[x ; \sigma]] g(x)=0$, completing the proof.

Let $\sigma$ be an endomorphism of a ring $R$ and $n \in \mathbb{N}$. An element $a$ of $R$ is called $\sigma(n)$-nilpotent if there exists a positive integer $m=m(n)$, depending on $n$, such that $a \sigma^{n}(a) \sigma^{2 n}(a) \cdots \sigma^{(m-1) n}(a)=0$. Equivalently, the element $a x^{n} \in R[x ; \sigma]$ is nilpotent.

Definition 2.5. Let $R$ be a ring and $\sigma$ an endomorphism of $R$.

(1) $N(\sigma ; i)(R):=\{a \in R \mid a$ is $\sigma(i)$-nilpotent $\}$.

(2) $N_{U}(\sigma)(R):=\cup_{i \in \mathbb{N}} N(\sigma ; i)(R)$.

(3) $N_{I}(\sigma)(R):=\cap_{i \in \mathbb{N}} N(\sigma ; i)(R)$.

Pearson and Stephenson [24] defined the $\sigma$-prime radical of a ring $R$, denoted by $P_{\sigma}(R)$, as the intersection of all strongly $\sigma$-prime ideals of $R$. Many authors have used the concept of $\sigma$-prime ideal to study of radicals of skew polynomial rings, see ([7], [13], [19], [23], [24], [25], [26], etc.). A subset $S$ of a ring $R$ is called $\sigma$-nil if every element in $S$ is $\sigma$-nilpotent. By [7, Proposition 1.12], $P_{\sigma}(R)$ is $\sigma$-nil. Note that $N_{U}(\sigma)(R)$ is the set of all $\sigma(n)$-nilpotent elements of $R$ and $N_{I}(\sigma)(R)$ is the set of all $\sigma$-nilpotent elements of $R$. Thus we see that $P_{\sigma}(R) \subseteq N_{I}(\sigma)(R) \subseteq N_{U}(\sigma)(R)$ when $\sigma$ is an automorphism. However, the following examples show that the containments are proper.

Example 2.6. (1) $P_{\sigma}(R) \subsetneq N_{I}(\sigma)(R)$.

Let $K\left[\left\{t_{i}\right\}_{i \in \mathbb{Z}}\right]$ be a polynomial ring over a field $K$, and $I=\left(\left\{t_{n_{1}} t_{n_{2}} t_{n_{3}} \mid n_{3}-\right.\right.$ $\left.\left.n_{2}=n_{2}-n_{1}>0\right\}\right)$ be an ideal of $K\left[\left\{t_{i}\right\}_{i \in \mathbb{Z}}\right]$. Define $R=K\left[\left\{t_{i}\right\}_{i \in \mathbb{Z}}\right] / I$. The $K$-automorphism $\sigma$ of $K\left[\left\{t_{i}\right\}_{i \in \mathbb{Z}}\right]$ defined by sending each $t_{i}$ to $t_{i+1}$ induces an automorphism $\sigma$ on $R$. Ram proved that the skew polynomial ring $R[x ; \sigma]$ is prime, see [26, Example 3.2(ii)]. Thus by [24, Corollary 1.4] we have that $P_{\sigma}(R)=0$. Note that for any integer $l \geq 1,\left(t_{i} x^{l}\right)^{3}=t_{i} \sigma^{l}\left(t_{i}\right) \sigma^{2 l}\left(t_{i}\right) x^{3 l}=$ $t_{i} t_{i+l} t_{i+2 l} x^{3 l}=0$. Hence $t_{i} \in N_{I}(\sigma)(R)$.

(2) $N_{I}(\sigma)(R) \subsetneq N_{U}(\sigma)(R)$.

Let $R=\mathbb{Z}_{2} \oplus \mathbb{Z}_{2}$ where $\mathbb{Z}_{2}$ is the ring of integers modulo 2 . Then $R$ is a commutative reduced ring. Consider $\sigma: R \rightarrow R$ the automorphism defined by $\sigma((a, b))=(b, a)$. Then $(1,0) \in N_{U}(\sigma)(R)$, but $N_{I}(\sigma)(R)=0$. Note that $R[x ; \sigma]$ is not reduced.

From now on, $\sigma$ always denotes an automorphism of a ring $R$. The following proposition extends the result of [28, Theorem 1.5].

Proposition 2.7. If $R$ is a $\sigma$-skew IFP ring, then $P_{\sigma}(R)=N_{I}(\sigma)(R)=$ $N_{U}(\sigma)(R)$. 
Proof. Let $a \in N_{U}(\sigma)(R)$. Then for some integer $s_{1} \geq 1$,

$$
a \sigma^{s_{1}}(a) \sigma^{2 s_{1}}(a) \cdots \sigma^{(m-1) s_{1}}(a)=0
$$

for some positive integers $m$. Assume that $a \notin P_{\sigma}(R)$. Then $a \notin P$ for some strongly $\sigma$-prime ideal of $R$ and by Lemma 1.10, we can take a positive integer $n_{1}>s_{1}$ such that $a R \sigma^{n_{1}}(a) \nsubseteq P$. Say $a x_{1} \sigma^{s_{1}}(a) \notin P$. Since $R$ is $\sigma$-skew IFP, we have

$$
a R \sigma^{n_{1}-s_{1}}\left(\sigma^{s_{1}}(a) \sigma^{2 s_{1}}(a) \cdots \sigma^{(m-1) s_{1}}(a)\right)=0 .
$$

In particular

$$
a x_{1} \sigma^{n_{1}}(a) \sigma^{n_{1}+s_{1}}(a) \cdots \sigma^{n_{1}+(m-2) s_{1}}(a)=0 .
$$

Also by Lemma 1.10, $a x_{1} \sigma^{n_{1}}(a) x_{2} \sigma^{n_{2}}(a) \notin P$ for some $x_{2} \in R$ and positive integer $n_{2}>n_{1}+s_{1}$. By the fact that $R$ is $\sigma$-skew IFP, we have that

$$
a x_{1} \sigma^{n_{1}}(a) R \sigma^{n_{2}-\left(n_{1}+s_{1}\right)}\left(\sigma^{n_{1}+s_{1}}(a) \cdots \sigma^{n_{1}+(m-2) s_{1}}(a)\right)=0
$$

and in particular we have that

$$
a x_{1} \sigma^{n_{1}}(a) x_{2} \sigma^{n_{2}}(a) \sigma^{n_{2}+s_{1}}(a) \cdots \sigma^{n_{2}+(m-3) s_{1}}(a)=0 .
$$

Next we can find $n_{3}>n_{2}+s_{1}$ and $x_{3} \in R$ such that

$$
a x_{1} \sigma^{n_{1}}(a) x_{2} \sigma^{n_{2}}(a) x_{3} \sigma^{n_{3}}(a) \notin P .
$$

Continuing in this process, we inductively obtain

$$
a x_{1} \sigma^{n_{1}}(a) x_{2} \sigma^{n_{2}}(a) x_{3} \sigma^{n_{3}}(a) \cdots x_{m-1} \sigma^{n_{m-1}}(a) \notin P
$$

and

$$
a x_{1} \sigma^{n_{1}}(a) x_{2} \sigma^{n_{2}}(a) x_{3} \sigma^{n_{3}}(a) \cdots x_{m-1} \sigma^{n_{m-1}}(a)=0
$$

for some $x_{j}$ 's in $R$, a contradiction. So $P_{\sigma}(R)=N_{I}(\sigma)(R)=N_{U}(\sigma)(R)$.

Lemma 2.8. If $R$ is a $\sigma$-skew IFP ring with $\sigma$ of locally finite order, then for $a_{1}, a_{2}, \ldots, a_{t} \in R, a_{1} a_{2} \cdots a_{t}=0$ implies $\sigma^{k_{1}}\left(a_{1}\right) R \sigma^{k_{2}}\left(a_{2}\right) R \cdots R \sigma^{k_{t}}\left(a_{t}\right)=0$ for any nonnegative integers $k_{1}, k_{2}, \ldots, k_{t}$.

Proof. The proof will be done by induction on $t$ and we first assume $a_{1} a_{2}=0$ for $a_{1}, a_{2} \in R$. Then $\sigma^{k_{1}}\left(a_{1}\right) \sigma^{k_{1}}\left(a_{2}\right)=0$ for any positive integer $k_{1}$. Since $R$ is $\sigma$-skew IFP, then $\sigma^{k_{1}}\left(a_{1}\right) R \sigma^{n+k_{1}}\left(a_{2}\right)=0$ for any positive integer $n \geq 1$. Let $\sigma^{m_{2}}\left(a_{2}\right)=a_{2}$ and $k_{2}=n+k_{1}$. Then $k_{2}=p m_{2}+r$, where $0 \leq$ $r<m_{2}$. Take a positive integer $s$ such that $n=s m_{2}+\left(r-k_{1}\right) \geq 1$. Thus $\sigma^{k_{2}}\left(a_{2}\right)=\sigma^{n+k_{1}}\left(a_{2}\right)=\sigma^{s N+\left(r-k_{1}\right)+k_{1}}\left(a_{2}\right)=\sigma^{s N+r}\left(a_{2}\right)=\sigma^{r}\left(a_{2}\right)$ and we have that $\sigma^{k_{1}}\left(a_{1}\right) R \sigma^{k_{2}}\left(a_{2}\right)=0$ for any positive integer $k_{1}, k_{2}$. Now assume that it holds for $t-1$. Suppose that $a_{1} a_{2} \cdots a_{t}=0$ and by induction hypothesis, $\sigma^{k_{1}}\left(a_{1}\right) R \sigma^{k_{2}}\left(a_{2}\right) R \cdots R \sigma^{k_{t-1}}\left(a_{t-1}\right)=0$ for any positive integers $k_{1}, k_{2}, \ldots, k_{t-1}$. Hence $\sigma^{k_{1}}\left(a_{1}\right) R \sigma^{k_{2}}\left(a_{2}\right) R \cdots R \sigma^{k_{t-1}}\left(a_{t-1}\right) \sigma^{k_{t-1}}\left(a_{t}\right)=0$ and by the fact that $R$ is $\sigma$-skew IFP we have that

$$
\sigma^{k_{1}}\left(a_{1}\right) R \sigma^{k_{2}}\left(a_{2}\right) R \cdots R \sigma^{k_{t-1}}\left(a_{t-1}\right) R \sigma^{n+k_{t-1}}\left(a_{t}\right)=0
$$

for any positive integer $n \geq 1$. Let $k_{t}=n+k_{t-1}$ and $\sigma_{t}^{m}\left(a_{t}\right)=a^{t}$. Then $k_{t}=$ $q m_{t}+r$, where $0 \leq r<m_{t}$ and take a positive integer $u$ such that $n=u m_{t}+(r-$ 
$\left.k_{t-1}\right) \geq 1$. Consequently $\sigma^{k_{t}}\left(a_{t}\right)=\sigma^{n+k_{t-1}}\left(a_{t}\right)=\sigma^{u m_{t}+\left(r-k_{t-1}\right)+k_{t-1}}\left(a_{t}\right)=$ $\sigma^{u m_{t}+r}\left(a_{t}\right)=\sigma^{r}\left(a_{t}\right)$. So $\sigma^{k_{1}}\left(a_{1}\right) R \sigma^{k_{2}}\left(a_{2}\right) R \cdots R \sigma^{k_{t}}\left(a_{t}\right)=0$ for any positive integers $k_{1}, k_{2}, \ldots, k_{t}$.

The $\sigma$-Wedderburn radical of $R$, denoted by $W_{\sigma}(R)$, is given by

$$
W_{\sigma}(R)=\sum\{I \mid I \text { is a } \sigma \text {-nilpotent } \sigma \text {-ideal of } R\} \text {. }
$$

By [13, Lemma 3.1], $W_{\sigma}(R)=\left\{a \in R \mid \sum_{i=0}^{\infty} R \sigma^{i}(a) R\right.$ is $\sigma$-nilpotent $\}$. Note that for any automorphism $\sigma$ of $R, W_{\sigma}(R) \subseteq P_{\sigma}(R)$.

Proposition 2.9. If $R$ is a $\sigma$-skew IFP ring with $\sigma$ of locally finite order, then $W_{\sigma}(R)=P_{\sigma}(R)=N_{I}(\sigma)(R)=N_{U}(\sigma)(R)$.

Proof. By Proposition 2.7, it is enough to show that $W_{\sigma}(R) \supseteq P_{\sigma}(R)$. Let $a \in P_{\sigma}(R)$ and $A=\sum_{i=0}^{\infty} R \sigma^{i}(a) R=\sum_{i=0}^{k-1} R \sigma^{i}(a) R$, where $\sigma^{k}(a)=a$. Then for any integer $s \geq 1$, there exists a positive integer $n=n(s)$ such that $a \sigma^{s}(a) \sigma^{2 s}(a) \cdots \sigma^{(n-1) s}(a)=0$. Since $R$ is $\sigma$-skew IFP, we have, by Lemma 2.8 , that $\sigma^{k_{1}}(a) R \sigma^{k_{2}}\left(\sigma^{s}(a)\right) R \cdots R \sigma^{k_{n}}\left(\sigma^{(n-1) s}(a)\right)=0$ for any positive integers $k_{1}, k_{2}, \ldots, k_{n}$. For $\sigma^{l_{1}}(a), \sigma^{l_{2}}(a), \ldots, \sigma^{l_{n}}(a) \in A$, take $k_{1}=l_{1}, \ldots, k_{n}=l_{n}$ and we have that

$$
\begin{aligned}
& \sigma^{l_{1}}(a) R \sigma^{s}\left(\sigma^{l_{2}}(a)\right) R \cdots R \sigma^{(n-1) s}\left(\sigma^{l_{n}}(a)\right) \\
= & \sigma^{k_{1}}(a) R \sigma^{s}\left(\sigma^{k_{2}}(a)\right) R \cdots R \sigma^{(n-1) s}\left(\sigma^{k_{n}}(a)\right) \\
= & \sigma^{k_{1}}(a) R \sigma^{k_{2}}\left(\sigma^{s}(a)\right) R \cdots R \sigma^{k_{n}}\left(\sigma^{(n-1) s}(a)\right) \\
= & 0 .
\end{aligned}
$$

So $A$ is a $\sigma$-nilpotent $\sigma$-ideal, and it follows that $a \in A \subseteq W_{\sigma}(R)$.

It is clear that IFP rings are abelian, but the converse is not true. We also have the following result.

Proposition 2.10. Let $R$ be a $\sigma$-skew IFP ring. Then we have

(1) $e^{2}=e \in R$ if and only if $e \sigma(e)=\sigma(e) e=e$. In particular, $e=\sigma(e)$.

(2) $R$ is abelian.

(3) $R[[x ; \sigma]]$ is abelian.

Proof. (1) Let $e$ be an idempotent in $R$. Then $(e-1) e=0=e(e-1)$, and we have that $(e-1) \sigma(e)=0$. Thus, by assumption, $e \sigma(e-1)=0$ and it follows that $e \sigma(e)=\sigma(e)$ and $e \sigma(e)=e$ since $\sigma$ is an automorphism. Hence $e=\sigma(e)$ and consequently $\sigma(e) e=e^{2}=e$. Suppose that $e \sigma(e)=\sigma(e) e=e$ for $e \in R$. Then $(1-\sigma(e)) e=0$, and by assumption $(1-\sigma(e)) \sigma(e)=0$. So, $e^{2}=e$.

(2) Let $e^{2}=e \in R$. Then $(1-e) e=0=e(1-e)$. By Lemma 1.4(2) and $(1)$, we have $0=(1-e) R \sigma(e)=(1-e) R e$ and $0=e R \sigma(1-e)=e R(1-e)$. So, $R$ is abelian.

(3) Note that if $e^{2}=e \in R[[x ; \sigma]]$, then $e \in R$ by the same method as in the proof of $[12$, Lemma 19]. Using (1) and (2), we get $R[[x ; \sigma]]$ is abelian. 
Finally, we consider the classical right quotient rings of skew power-serieswise Armendariz ring and skew IFP rings. Recall that a ring $R$ is right Ore if there exists the classical right quotient $\operatorname{ring} Q(R)$ of $R$. Let $\sigma$ be an automorphism of a right Ore ring $R$. Then for any $a b^{-1} \in Q(R)$ where $a, b \in R$ with $b$ regular, the induced map $\bar{\sigma}: Q(R) \rightarrow Q(R)$ defined by $\bar{\sigma}\left(a b^{-1}\right)=\sigma(a) \sigma(b)^{-1}$ extends to an automorphism of $Q(R)$.

Proposition 2.11. Suppose that $R$ is a semiprime right Ore ring. Then the following statements are equivalent:

(1) $R$ is $\sigma$-rigid.

(2) $R$ is $\sigma$-skew power-serisewise Armendariz.

(3) $R$ is $\sigma$-skew IFP.

(4) $Q(R)$ is $\bar{\sigma}$-rigid.

(5) $Q(R)$ is $\bar{\sigma}$-skew power-serisewise Armendariz.

(6) $Q(R)$ is $\bar{\sigma}$-skew IFP.

Proof. The directions $(1) \Rightarrow(2) \Rightarrow(3)$ and $(4) \Rightarrow(5) \Rightarrow(6)$ follow from Theorem 1.1 and Lemma $1.4(2)$. It is easy to show $(1) \Leftrightarrow(4)$. $(6) \Rightarrow(4)$ : Suppose $a b^{-1} \bar{\sigma}\left(a b^{-1}\right)$ $=0$ for $a b^{-1} \in Q(R)$. Then

$$
a b^{-1} \sigma(a)=0 \Rightarrow a b^{-1} Q(R) \sigma^{2}(a)=0 \Rightarrow a Q(R) \sigma^{2}(a)=0 \Rightarrow a R \sigma^{2}(a)=0 .
$$

Since $R$ is semiprime we have that

$\sigma^{2}(a) a=0 \Rightarrow \sigma^{2}(a) Q(R) \sigma^{2}(a)=0 \Rightarrow \sigma^{2}(a) R \sigma^{2}(a)=0 \Rightarrow a R a=0 \Rightarrow a=0$.

By the same method as above, we also get the direction $(3) \Rightarrow(1)$.

Acknowledgments. The authors thank the referee for very careful reading of the manuscript and valuable suggestions that improved the paper. The first named author was supported by the Basic Science Research Program through the National Research Foundation of Korea(NRF) funded by the Ministry of Education(NRF-2011-0009313), the second named author was supported by Basic Science Research Program through the National Research Foundation of Korea(NRF) funded by the Ministry of Education(NRF-2013R1A1A4A010081 08), while the third named author was supported by the Research Fund Program of Research Institute for Basic Sciences, Pusan National University, Korea, 2015, Project No. RIBS-PNU-2015-101.

\section{References}

[1] R. Antoine, Nilpotent elements and Armendariz rings, J. Algebra 319 (2008), no. 8, 3128-3140.

[2] E. P. Armendariz, A note on extensions of Baer and P.P.-rings, J. Austral. Math. Soc. 18 (1974), 470-473.

[3] M. Baser, A. Harmanci, and T. K. Kwak, Generalized semicommutative rings and their extensions, Bull. Korean Math. Soc. 45 (2008), no. 2, 285-297.

[4] H. E. Bell, Near-rings in which each element is a power of itself, Bull. Austral. Math. Soc. 2 (1970), 363-368. 
[5] G. M. Bergman, Modules over coproducts of rings, Trans. Amer. Math. Soc. 200 (1974), $1-32$.

[6] Coproducts and some universal ring constructions, Trans. Amer. Math. Soc. 200 (1974), 33-88.

[7] J. S. Cheon, E. J. Kim, C. I. Lee, and Y. H. Shin, Characterizations of elements in prime radicals of skew polynomial rings and skew Laurent polynomial rings, Bull. Korean Math. Soc. 48 (2011), no. 2, 277-290.

[8] W. Cortes, Skew Armendariz rings and annihilator ideals of skew polynomial rings, Algebraic structures and their representations, 249259, Contemp. Math., 376, Amer. Math. Soc., Providence, RI, 2005.

[9] _ Skew polynomial extensions over zip rings, Int. J. Math. Math. Sci. (2008), Art. ID 496720, 9 pp.

[10] D. E. Fields, Zero divisors and nilpotent elements in power series rings, Proc. Amer. Math. Soc. 22 (1971), 427-433.

[11] E. Hamann and R. G. Swan, Two counterexamples in power series rings, J. Algebra 100 (1986), no. 1, 260-264.

[12] C. Y. Hong, N. K. Kim, and T. K. Kwak, On skew Armendariz rings, Comm. Algebra 31 (2003), no. 1, 103-122.

[13] C. Y. Hong, N. K. Kim, and Y. Lee, Radicals of skew polynomial rings and skew Laurent polynomial rings, J. Algebra 331 (2011), 428-448.

[14] C. Y. Hong, T. K. Kwak, and S. T. Rizvi, Extensions of generalized Armendariz rings, Algebra Colloq. 13 (2006), no. 2, 253-266.

[15] C. Huh, Y. Lee, and A. Smoktunowicz, Armendariz rings and semicommutative rings, Comm. Algebra 30 (2002), no. 2, 751-761.

[16] N. K. Kim, K. H. Lee, and Y. Lee, Power series rings satisfying a zero divisor property, Comm. Algebra 34 (2006), no. 6, 2205-2218.

[17] J. Krempa, Some examples of reduced rings, Algebra Colloq. 3 (1996), no. 4, 289-300

[18] T. Y. Lam, Lectures on Modules and Rings, Springer-Verlag, New York, 1999.

[19] T. Y. Lam, A. Leroy, and J. Matcsuk, Primeness, semiprimeness and prime radical of Ore extensions, Comm. Algebra 25 (1997), no. 8, 2459-2506.

[20] G. Marks, Skew polynomial rings over 2-primal rings, Comm. Algebra 27 (1999), no. 9, 4411-4423.

[21] J. Matczuk, A characterization of $\sigma$-rigid rings, Comm. Algebra 32 (2004), no. 11, 4333-4336.

[22] L. Motais de Narbonne, Anneaux semi-commutatifs et unis riels anneaux dont les id aux principaux sont idempotents, Proceedings of the 106th National Congress of Learned Societies (Perpignan, 1981), 71-73, Bib. Nat., Paris, 1982

[23] A. Moussavi, On the semiprimitivity of skew polynomial rings, Proc. Edinburgh Math. Soc. 36 (1993), no. 2, 169-178.

[24] K. R. Pearson and W. Stephenson, A skew polynomial ring over a Jacobson ring need not be a Jacobson ring, Comm. Algebra 5 (1977), no. 8, 783-794.

[25] K. R. Pearson, W. Stephenson, and J. F. Watters, Skew polynomials and Jacobson rings, Proc. London Math. Soc. (3) 42 (1981), no. 3, 559-576.

[26] J. Ram, On the semisimplicity of skew polynomial rings, Proc. Amer. Math. Soc. 90 (1984), no. 3, 347-351.

[27] M. B. Rege and S. Chhawchharia, Armendariz rings, Proc. Japan Acad. Ser. A Math. Sci. 73 (1997), no. 1, 14-17.

[28] G. Shin, Prime ideals and sheaf representation of a pseudo symmetric ring, Trans. Amer. Math. Soc. 184 (1973), 43-60. 
CHAN YONG HONG

Department of Mathematics and Research Institute for Basic Sciences KyUnG HeE UNIVERSiTy

SEOUl 131-701, KoreA

E-mail address: hcy@khu.ac.kr

NAM KYUN KIM

SCHOOL OF BASIC SCIENCES

Hanbat National University

DAEJEON 305-719, KOREA

E-mail address: nkkim@hanbat.ac.kr

YANG LEE

Department of Mathematics

Pusan National University

Pusan 609-735, Korea

E-mail address: ylee@pusan.ac.kr 